\title{
Radiotherapy-associated Furin Expression and Tumor Invasiveness in Recurrent Laryngeal Cancer
}

\author{
MYUNGJIN LEE ${ }^{1 *}$, CHANG HWAN RYU ${ }^{2}$, HYO WON CHANG ${ }^{1}$, GUI CHUL KIM ${ }^{1}$, \\ SEONG WHO KIM ${ }^{3}$ and SANG YOON KIM ${ }^{1,4}$ \\ Departments of ${ }^{1}$ Otolaryngology and ${ }^{3}$ Biochemistry and Molecular Biology, Asan Medical Center, \\ University of Ulsan College of Medicine, Seoul, Republic of Korea; \\ ${ }^{2}$ Department of Otolaryngology, Head and Neck Oncology Clinic, Center for Specific Organ Cancer and \\ Center for Thyroid Cancer, National Cancer Center, Goyang-si, Republic of Korea; \\ ${ }^{4}$ Biomedical Research Institute, Korea Institute of Science and Technology, Seoul, Republic of Korea
}

\begin{abstract}
Background/Aim: Recurrent laryngeal cancer often shows an aggressive phenotype after radiotherapy and does not respond to conventional therapeutic strategies. In this study, we investigated the contribution of furin to cellular invasiveness in radio-resistant laryngeal cancer. Materials and Methods: Using previously established AMC$H N-3$ and $A M C-H N-8$ cell lines from laryngeal carcinoma patients, recurrent laryngeal cancer models were generated by cumulative irradiation (AMC-HN-3-70Gy and AMC-HN8-70Gy). Immunocytochemistry and western blotting were used to determine the epithelial-mesenchymal transition (EMT). Invasion capacity was assessed using an in vitro invasion assay. Zymography was used to assess metalloproteinase-2 (MMP-2) activity. Tumor xenografts were developed to compare growth rate and furin expression in vivo. Furin expression in 35 patients (45 samples) with salvage total laryngectomy after radiation-based treatment was assessed by laryngeal cancer tissue microarray. Results: Both AMC-HN-3-70Gy and AMC-HN-8-70Gy cell
\end{abstract}

\footnotetext{
*These Authors contributed equally to this study.

Correspondence to: Sang Yoon Kim, Department of Otolaryngology, Asan Medical Center, University of Ulsan College of Medicine, 86 Asanbyeongwon-gil, Songpa-gu, Seoul 138-736 and Biomedical Research Institute, Korea Institute of Science and Technology, Hwarangno 14-gil 5, Seongbuk-gu, Seoul, 136-791, Republic of Korea. Tel: +82 20103715, Fax: +82 24892773, e-mail: sykim2@amc.seoul.kr and Seong Who Kim, Department of Biochemistry and Molecular biology, Asan Medical Center, University of Ulsan College of Medicine, 88, Olympic-Ro 43-Gil, Songpa-Gu, Seoul 138-736, Republic of Korea. Tel: +82 230104270, Fax: +82 230108098, e-mail: swhokim@amc.seoul.kr
}

Key Words: Radiotherapy, furin, MT1-MMP, MMP-2, invasion, laryngeal cancer. lines underwent EMT following radiation. However, AMC$H N-3-70 G y$ cells showed increased cellular invasiveness, whereas AMC-HN-8-70Gy cells showed no difference. AMC-HN-3-70Gy cells also exhibited elevated furin expression with up-regulated expression of the active form of membrane type 1-matrix metalloproteinase (MT1$M M P) / M M P-2$, whereas AMC-HN-8-70Gy cells did not show significant changes. After administration of a furin inhibitor (chloromethyl ketone (CMK)), AMC-HN-3-70Gy cells showed a significant decrease in MT1-MMP/MMP-2 expression and cellular invasiveness. Nine of 22 samples (40.9\%) from salvage total laryngectomy and one of 13 preradiation samples (7.7\%) had high furin expression. Postradiation, furin expression increased in seven of 10 patients whose pre-and post-radiation samples were available; allcancer mortality (three patients) was observed in this group. Conclusion: Together with EMT, furin activity may serve as an indicator of an aggressive cancer phenotype, suggesting that furin is a potentially useful target for recurrent laryngeal cancer.

Classically, advanced laryngeal cancer is treated with surgery. However, surgical resection of this region sometimes leaves the patient with a reduced quality of life owing to the severe loss of laryngeal functions, such as respiration, swallowing and phonation (1). Therefore, traditional approaches to management of laryngeal cancer have been modified to reduce treatment-related morbidity and improve the patient's quality of life (1-3).

Radiotherapy plays an essential role in the treatment of laryngeal cancer. Early laryngeal cancer can be efficiently cured with radiotherapy alone. Radiation-based therapy with concurrent or sequential chemotherapy is now the mainstay of treatment of advanced laryngeal cancer. These efforts have gradually increased the survival of laryngeal cancer patients and minimized the reduction in quality of 
life. However, nearly $50 \%$ of patients present with locoregional relapse in previously irradiated tissues within 2 years (1-3).

Recurrent laryngeal cancer is very often difficult to treat with curative intent. Currently, treatment options for these patients include salvage surgery, re-irradiation with or without chemotherapy, or chemotherapy only. Unfortunately, re-irradiation of recurrent laryngeal cancer is limited because of normal tissue tolerance. Surgery is often the treatment of choice; however, it is feasible for only a small subset $(<15 \%)$ of patients $(4,5)$. Current chemotherapy, including newer molecular therapies targeting epidermal growth factor (EGF) or vascular endothelial growth factor (VEGF), does not show promising results $(6,7)$.

Targeting mechanisms of radio-resistance seems to be a logical approach for treating laryngeal cancer that recurs after radiation-based treatment. To this end, we have recently developed recurrent laryngeal cancer cell lines resistant to clinically relevant doses of irradiation. These radio-resistant cell lines show different biological characteristics as compared to their parent cell lines, suggesting they are appropriate for investigating the mechanisms involving radiation-induced molecular changes (8). Using these cells, we investigate new targets for treating radio-resistant laryngeal cancer.

Within the Golgi/trans-Golgi network secretory pathway, the furin/proprotein convertases catalyze the activation or functionalization of inactive precursor proteins by processing the specific recognition motif (RX/RR). Furin further activates many precursors that are not only vital for proper physiological function but are also responsible for several pathological conditions, including degenerative diseases, arthritis and cancer. Furin expression and processing have been implicated in cancer development and progression (9-11).

Acquisition of the invasive phenotype is a crucial element of tumor progression. Metalloproteinase-2 (MMP-2), a key molecule in cancer invasion and metastasis, is produced in a pro-form and is activated by membrane type 1-matrix metalloproteinase (MT1-MMP). MT1-MMP is activated by furin intracellularly before secretion in the Golgi complex (9, 11-14). Although furin and MMP-2 expressions are associated with aggressive phenotypes in several types of cancer (9-11, 15-17), their mechanistic association with the aggressiveness of recurrent cancers following irradiation remains under investigation.

In this study, we investigated the contribution of furin to cellular invasiveness of chronic, cumulatively irradiated laryngeal cancer cells. We reveal that furin may be a good biomarker for aggressiveness and a potential target for treating radio-resistant laryngeal cancer. Exploration of furinmediated invasion mechanisms could expedite the development of new anticancer targeted modalities.

\section{Materials and Methods}

Cell culture and the establishment of recurrent laryngeal cancer cell lines after irradiation. The human head and neck cancer cell lines (AMC-HN-3 and AMC-HN-8) were previously established from squamous cell carcinoma of the larynx (18). Both cancer cells were cultured in $\alpha$-MEM media (Invitrogen, Grand Island, NY, USA), supplemented with $2 \mathrm{mM}$ L-glutamine, $1 \%$ nonessential amino acids, $100 \mathrm{U} / \mathrm{ml}$ penicillin, $100 \mu \mathrm{g} / \mathrm{ml}$ streptomycin and $10 \%$ heat inactivated fetal bovine serum ((FBS); Invitrogen) and maintained at $37^{\circ} \mathrm{C}$ in a humidified atmosphere with $5 \% \mathrm{CO}_{2}$. When the cells had grown to approximately $70-80 \%$ confluence in a $100-\mathrm{mm}^{2}$ dish, they were irradiated using 6-MV photon beam generated by a linear accelerator (CLINAC600C; Varian Co., Milpitas, CA, USA) at a dose of $2 \mathrm{~Gy}$. On reaching 90\% confluence, the cells were trypsinized and subcultured. This procedure was repeated until the cells had received a cumulative dose of $70 \mathrm{~Gy}$, after which an isogenic models of successively radioresistant cells (AMC-HN-370Gy and AMC-HN-8-70Gy) were considered established. For all experiments with irradiated cells, at least a 10-day recovery period after the last 2 Gy irradiation was allowed (19).

Immunocytochemistry. Cells were grown on coverslips in 24-well plates (Nunc, Roskilde, Denmark) and fixed with $3.7 \%$ paraformaldehyde for $15 \mathrm{~min}$ at room temperature, followed by treatment with $0.1 \%$ Triton for $20 \mathrm{~min}$. The samples were washed three times with PBS, blocked with $1 \%$ skim milk at room temperature for $1 \mathrm{~h}$ and incubated with primary antibodies E-cadherin, snail, fibronectin (Santa Cruz Biotechnology, Santa Cruz, CA, USA) and vimentin (DAKO, Glostrup, Denmark) for $2 \mathrm{~h}$ at room temperature or overnight at $4^{\circ} \mathrm{C}$. After this step, the cells were washed with PBS and incubated with fluorescein isothiocyanate (FITC) or rhodamineconjugated secondary antibodies (Jackson ImmunoResearch, West Grove, PA, USA) at room temperature in dark for $1 \mathrm{~h}$. Nuclei were counterstained with 4',6-diamidino-2-phenylindole (DAPI) for $5 \mathrm{~min}$. After washing three times with PBS, the cells were mounted with DAKO fluorescence mounting solution and observed by laser confocal microscopy (Leica, St. Gallen, Switzerland).

Western blot analysis. After reaching $80-90 \%$ confluence in a $100-\mathrm{mm}^{2}$ dish (Nunc, Roskilde, Denmark), the cells were washed with PBS, collected with scratcher, lysed with ProPrep protein extraction solution (iNtRON Biotechnology, Sung-Nam, Korea) and centrifuged at $1,500 \mathrm{rpm}$ for $3 \mathrm{~min}$. Supernatants were collected and the total protein was determined using the Bradford protein assay kit (Bio-Rad Laboratories, Hercules, CA, USA). The extracted proteins ( $20 \mu \mathrm{g}$ per lane) were fractionated by $7.5 \%-12 \%$ SDS-polyacrylamide gel electrophoresis and transferred to Hybond-PVDF membranes (Amersham International PLC, Buckinghamshire, UK). After blocking with Tris-buffered saline with Tween 20 (TBST) buffer containing 5\% BSA for $30 \mathrm{~min}$ at room temperature, the membranes were incubated with primary antibodies against E-cadherin, snail, fibronectin, furin, MT1-MMP (Santa Cruz Biotechnology) and vimentin (DAKO) overnight at $4^{\circ} \mathrm{C}$. Membranes were washed three times with TBST and incubated with the secondary antibody (anti-rabbit IgG horseradish peroxidase (HRP) conjugate; Zymed, San Francisco, CA, USA) for $1 \mathrm{~h}$ at room temperature. After washing, the specific binding of the antibodies was detected using SuperSignal West Pico Trial kit (Thermo Scientific, Rockford, IL, USA) according to the 
manufacturer's instructions. Films were exposed at multiple time points to ensure that the images were not saturated.

Invasion assay. Invasive ability of the cells was assessed with Biocoat collagen invasion chambers (Chemicon, Temecular, CA, USA). The cells were trypsinized and 10,000 cells were suspended in serum-free alpha-smooth muscle actin ( $\alpha$-SMA) with $2 \%$ FBS. In the inhibition studies, $50 \mu \mathrm{M}$ CMK (R\&D Systems, Inc., Minneapolis, MN, USA) was added to this medium. Medium containing 5\% serum was used as chemoattractant. Incubation was performed for $22 \mathrm{~h}$. Cells that degraded the reconstituted extracellular matrix (ECM) and passed through the invasion chamber were fixed.

Furin activity assay. To compare the expression levels of furin in AMC-HN-3 and HN-3-70Gy cells, an equal number of cells from each cell line was plated and cultured for 24 hours in reduced serum (2\% FBS) media. The cells were then collected for ELISA assays.

Gelatin zymography. A total of $5 \times 10^{6}$ cells were grown overnight in serum-free $\alpha$-MEM with $2 \mathrm{mmol} / \mathrm{l} \mathrm{L}$-glutamine. The conditioned media were concentrated to $200 \mu \mathrm{l}$ by ultrafiltration (Centriprep 30, Amicon Millipore, Bedford, MA, USA) and $30 \mu \mathrm{g}$ were loaded in a $10 \%$ SDS gel containing $1 \%$ gelatin. After electrophoresis, the gels were washed tree times in $200 \mathrm{ml}$ of $2.5 \%$ Triton X-100 to remove SDS and renature the MMP-2/9 species in the gels. Then the gels were incubated overnight at $37^{\circ} \mathrm{C}$ in $0.05 \mathrm{M}$ Tris- $\mathrm{HCl}$ buffer, $\mathrm{pH} 7.5$, containing $10 \mathrm{mM} \mathrm{CaCl}$ to induce gelatin lysis by renatured MMP$2 / 9$. The gels were stained with $0.25 \%$ Coomassie brilliant blue R-250 (50\% methanol, $10 \%$ acetic acid) and de-stained in methanol:acetic acid: water (50:10:40). Clear zones indicated the presence of gelatinolytic activity in zymography, whereas dark zones indicated the presence of gelatinase inhibitors in reverse zymography. Zymography standards were purchased from Chemicon (Temecula, CA, USA).

Immunohistochemistry of tumor xenografts. Male athymic BALB/c $\mathrm{nu} / \mathrm{nu}$ nude mice ( 6 weeks of age) were purchased from ORIENT BIO Inc. (Seongnam, Korea). The mice were maintained in a pathogen-free facility and all animal studies were performed according to the protocol approved by the Institutional Animal Care and Use Committee (IACUC) at the Laboratory of Animal Research, Asan Institute for Life Sciences (Asan Medical Center, Seoul, Korea). To evaluate the expression of furin in tumor xenograft, $1 \times 10^{7}$ AMC HN-3 or HN-3-70Gy cells were subcutaneously injected into the right flank of the mice. Caliper measurements were performed every other day and the tumor volume was calculated as $\mathrm{D} \times \mathrm{d} \times$ height $\times 0.52$, where $\mathrm{D}=$ the long diameter and $\mathrm{d}=$ the perpendicular short diameter. Tumor xenografts were harvested and pariffinzed. Deparaffinization and hydration of $4-\mu \mathrm{m}$ thick sections were followed by heat-induced antigen retrieval using a $0.01 \mathrm{M}$ citrate buffer ( $\mathrm{pH}$ 6.0) for $1 \mathrm{~h}$. Sections were incubated in aqueous $3 \% \mathrm{H}_{2} \mathrm{O}_{2}$ for 15 min to quench endogenous peroxidase activity and washed with $1 \times$ PBS. Slides were loaded onto a humid chamber and blocked for 30 min with $1 \times$ Universal Blocking Agent (10× Power Block ${ }^{\mathrm{TM}}$; BioGenex, San Ramon, CA, USA) and then incubated in the primary antibody against furin (1:200; Santa Cruz) overnight at $4^{\circ} \mathrm{C}$. The next day, slides were incubated for $1 \mathrm{~h}$ at room temperature and treated with Envision Reagent (Dako REAL ${ }^{\mathrm{TM}}$ EnVision $^{\mathrm{TM}}$; DAKO) for $30 \mathrm{~min}$. The resulting product was washed with $\mathrm{PBS}$ and treated with the chromogen 3,3'-diaminobenzidine (DAB) for $15 \mathrm{~min}$ forming a brown reaction product. The slides were then counterstained in Mayer's hematoxylin, dehydrated in graded alcohol, cleared in xylene and mounted on the slide.

Patients and tissue samples. We previously constructed tissue microarray (TMA) with 111 specimens from 100 patients with laryngeal cancer, diagnosed between 1997 and 2011, and retrieved from Asan Medical Center. Duplicate 0.6-mm tissue cores were used to construct the TMAs with an ATA-27 automated tissue arrayer (Estigen, Tartu, Estonia). From these TMA samples, we could identify 35 patients (45 samples) with salvage total laryngectomy after radiation-based therapy. We analyzed the furin staining status (Santa Cruz Biotechnology) in these patients. Array blocks were sectioned to produce serial $4-\mu \mathrm{m}$ sections and the first section was stained with $H \& E$ to assess adequacy. The remaining sections were stored at room temperature before immunostaining. The use of the tissue samples was approved by the Institutional Review Board of Asan Medical Center.

Statistical analysis. Student's $t$-test was used for the analysis of invasion and furin activity assays. Chi-square test for descriptive variables and non-parametric Wilcoxon signed rank test were used for patient samples. A value of $p<0.05$ was considered statistically significant.

\section{Results}

Laryngeal cancer cells show ionizing radiation-induced morphological and molecular changes consistent with epithelial-mesenchymal transition (EMT), but different invasion potentials. Laryngeal cancer cells (AMC-HN-3 and AMC-HN-8), which were continuously irradiated up to $70 \mathrm{~Gy}$ in 2 Gy intervals every 2-3 days, showed markedly different morphological and molecular changes compared to their parental cell lines. Both types of cells lost their epithelial morphology and acquired mesenchymal traits. Cells appeared dispersed and formed a spindle-cell appearance consistent with EMT, as shown in Figure 1A. In AMC-HN-3-70Gy cells, these morphological changes were accompanied by upregulated expression of mesenchymal markers, such as snail, vimentin and fibronectin, as detected by western blot and immunocytochemistry. AMC-HN-8-70Gy cells showed increased expression of fibronectin and vimentin in immunocytochemistry and western blot analyses but did not show substantial changes in snail expression. Membranous Ecadherin expression was decreased in AMC-HN-3-70Gy cells, while expression of E-cadherin was unchanged in AMC-HN-8-70Gy cells after radiation (Figure 1B and C).

Despite different mesenchymal marker expression profiles, both types of laryngeal cancer cells shifted to EMT after irradiation, showing mesenchymal morphology and molecular changes. However, each laryngeal cancer cell line showed different invasion potential after the shift to EMT. AMC-HN3-70Gy cells showed increased cellular invasiveness than AMC-HN-3 cells $(p=0.037)$. However, AMC-HN-8-70Gy cells showed reduced invasion capacity than their parent cells (Figure 1D). 

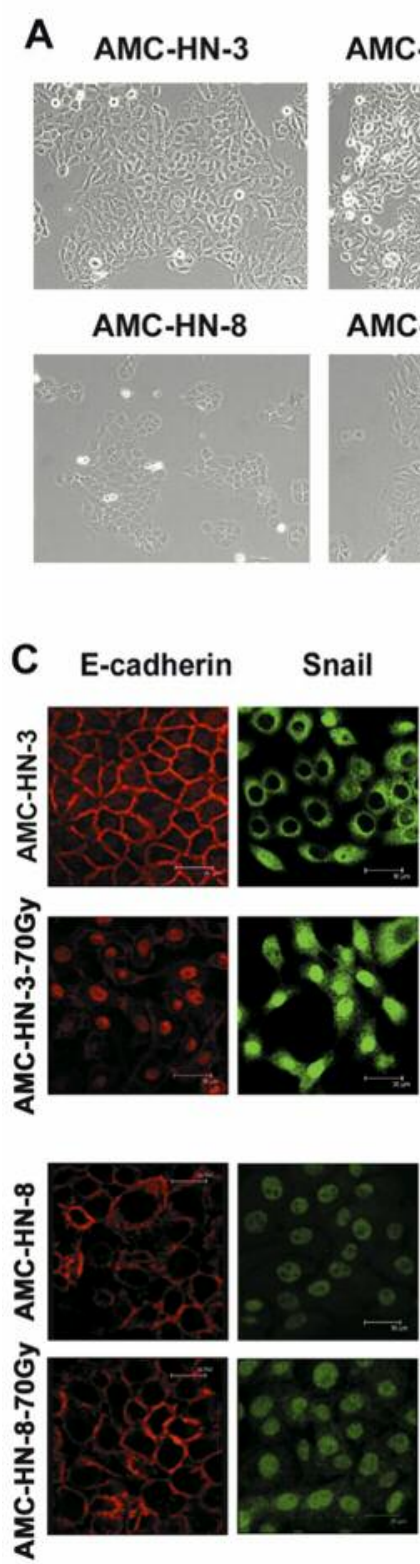

AMC-HN-3-70Gy

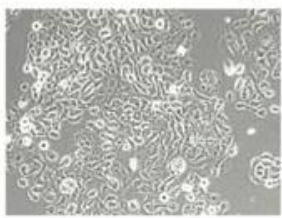

AMC-HN-8-70Gy

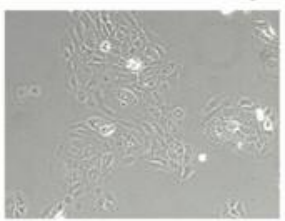

B

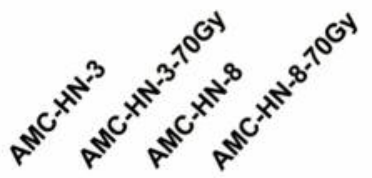

\section{E-cadherin}

Snail

Vimentin

Fibronectin

$\beta$-actin
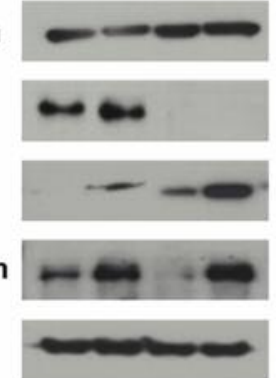

Fibronectin
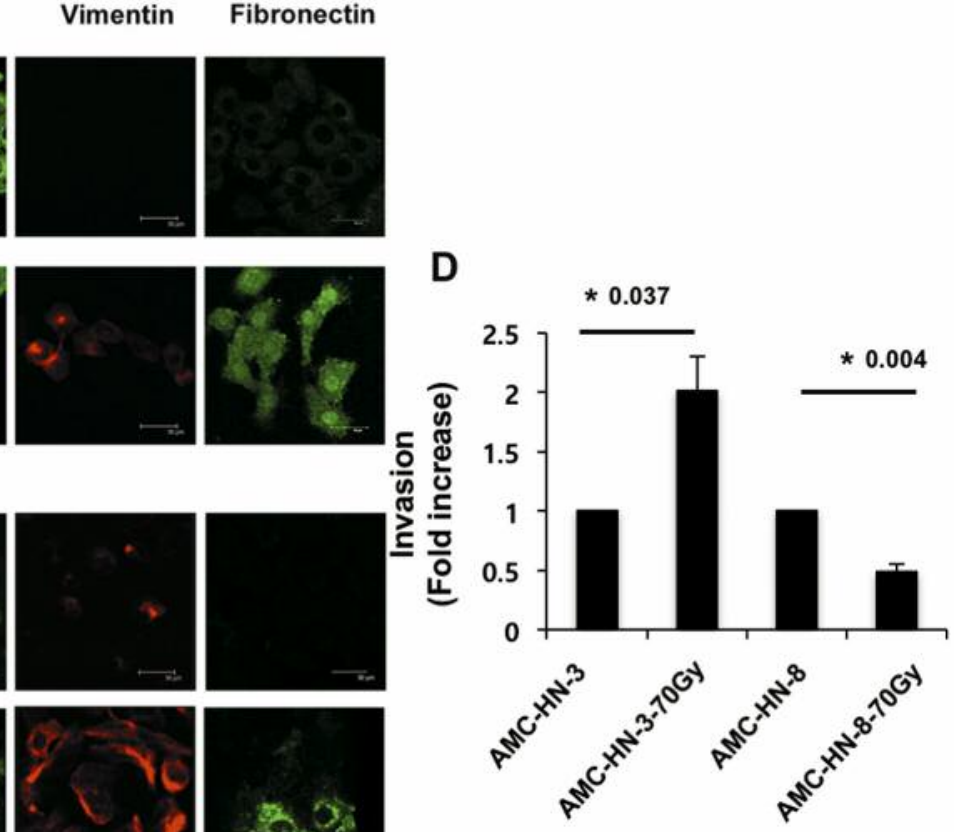

Figure 1. Epithelial-mesenchymal transition (EMT) of laryngeal cancer cells after repetitive irradiation (70 Gy) shows different invasion potentials. (A) Phase-contrast images of laryngeal cancer cells before and after repetitive irradiation. After irradiation, both AMC-HN-3-70Gy and AMC-HN8-70Gy cells exhibited a spindle-cell appearance and loss of epithelial morphology. (B) Western blot analysis of expression of E-cadherin, snail, vimentin and fibronectin in cells described in (A). $\beta$-actin was used as a loading control. (C) Images of cells described in (A) immunostained with antibodies against E-cadherin, snail, vimentin and fibronectin. AMC-HN-3-70Gy cells showed an increased expression of snail and fibronectin. Vimentin was weakly positive by immunocytochemistry. AMC-HN-8-70Gy cells showed increased expression of fibronectin and vimentin, but not of snail, after irradiation. E-cadherin expression (membranous) was decreased in AMC-HN3-70Gy. (D) Invasion assays of cells described in (A). $A M C-H N-3-70 G y$ cells showed increased cellular invasiveness compared to AMC-HN-3 cells.

Radiation-induced cellular invasion by MT1-MMP and MMP2 activation is controlled by furin. To investigate the different invasion potentials in the two laryngeal cancer cell lines, we examined MT1-MMP and MMP-2/9 expression and activity.
Consistent with the invasion assay, protein expression of active MT1-MMP and MMP-2 was increased in AMC-HN-370Gy cells, unlike in AMC-HN-8-70Gy cells, compared to their parent cells (Figure 2A). Furin activity was significantly 


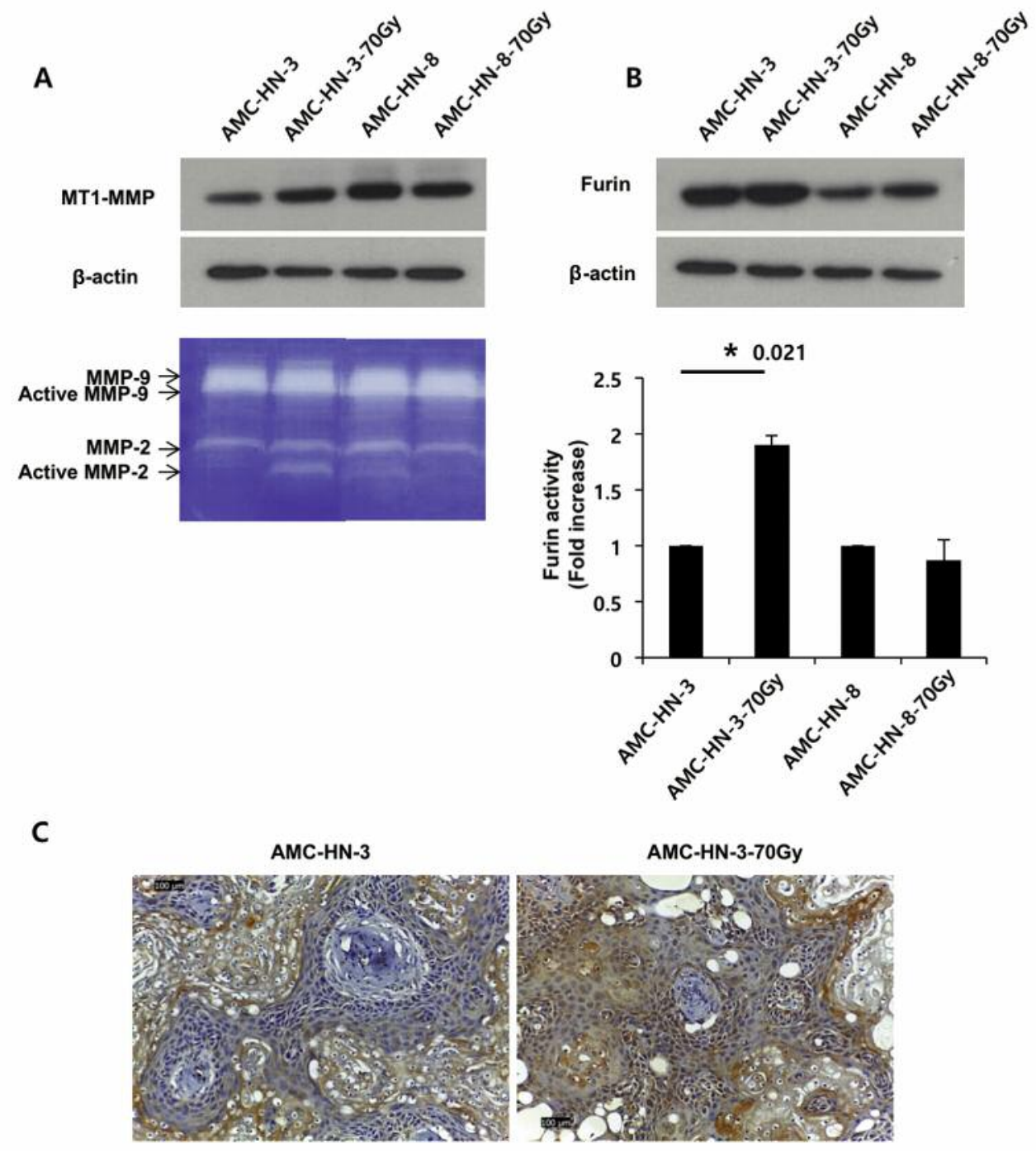

Figure 2. Radiation-induced cellular invasion of laryngeal cancer cells is associated with increased expression of the active form of MT1MMP/MMP-2 and furin activity. (A) Protein levels of MT1-MMP and the activities of MMP-2/9 in laryngeal cancer cells before and after repetitive irradiation as measured by western blot and gelatin zymography, respectively. Active MT1-MMP and MMP-2 expression was increased in AMCHN-3-70Gy cells, unlike in AMC-HN-8-70Gy cells. (B) Furin activity of cells described in (A) by western blot (upper) and ELISA (lower). Furin expression and activity were significantly increased in AMC-HN-3-70Gy cells compared to AMC-HN-3 cells. Data are reported as mean $\pm S E M$. (C) Immunohistochemical staining of furin in AMC-HN-3 and AMC-HN-3-70Gy xenografts. AMC-HN-3-70Gy cells showed higher expression of furin than AMC HN-3 cells.

increased in AMC-HN-3-70Gy cells compared to AMC-HN3 cells, while AMC-HN-8 cells did not show a substantial change in furin activity after chronic cumulative irradiation (Figure 2B). AMC-HN-3-70Gy cells showed stronger expression of furin than AMC-HN-3 cells (Figure 2C).

On treatment with a furin inhibitor (CMK), AMC-HN-370Gy cells showed decreased active MMP-2 and MT1-MMP expression, unlike AMC-HN-8-70Gy cells, compared to their parent cells (Figure 3A). Treatment of AMC-HN-3-70Gy cells with the furin inhibitor CMK decreased cellular invasiveness, whereas AMC-HN-8-70Gy cells did not show substantial changes (Figure 3B). To confirm that furin expression is increased by cumulative irradiation, we conducted furin expression analysis using a tissue microarray of 45 samples from 35 patients with recurrent laryngeal squamous cell carcinoma after radiation-based therapy (Figure 4A-C). Of the 35 patients who underwent salvage total laryngectomy, samples from 22 patients and 3 patients were obtained during salvage laryngectomy and pre-radiation, respectively; for 10 patients, both pre- and post-radiation samples were obtained. 


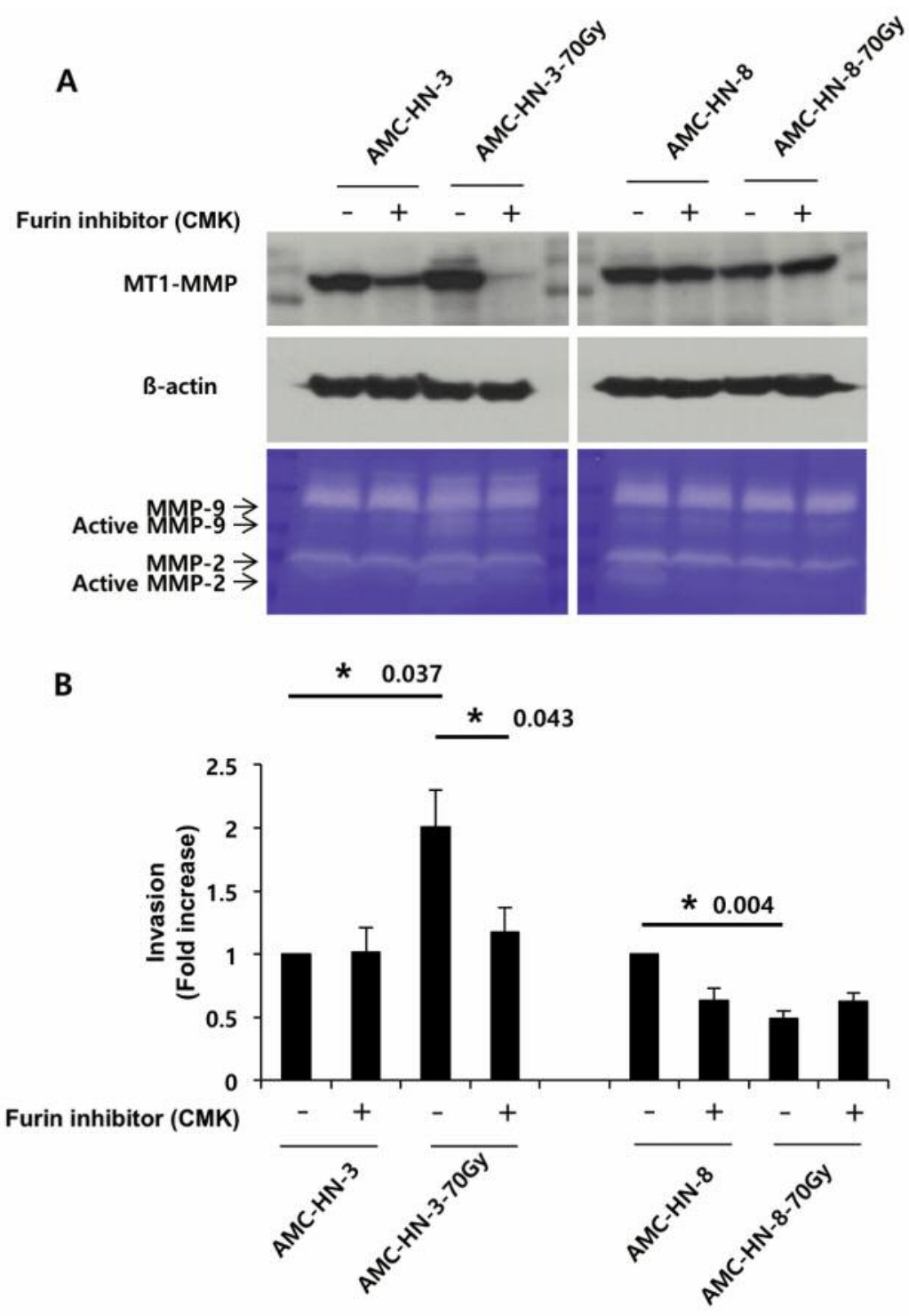

Figure 3. Radiation-induced cellular invasion of laryngeal cancer cells is controlled by furin. (A) Protein levels of active MT1-MMP and MMP-2/9 in laryngeal cancer cells treated with furin inhibitor, chloromethyl ketone $(C M K)$, before and after repetitive irradiation as measured by western blot and gelatin zymography, respectively. (B) Invasion assays of cells described in (A) treated with furin inhibitor. In the presence of furin inhibitor, active MT1-MMP and MMP-2 expression levels decreased with corresponding changes in cellular invasion. Cellular invasion decreased in AMCHN3-70Gy in the presence of furin inhibitor.

Table I describes the clinical characteristics of these patients. Furin IHC staining was mostly cytoplasmic but, in some patients, membrane accentuation was noted. From the 22 salvage total laryngectomy samples, 9 (40.9\%) specimens had high furin expression; of the 13 pre-radiation samples, only 1 sample $(7.7 \%)$ showed high furin expression (Figure 4D, $p=0.036$ ). Of the 10 patients with both pre- and post-radiation samples collected, furin expression was increased in 7 patients after radiation; all cancer mortality ( 3 patients) was observed in this group (Table II, Figure 4E-H, $p=0.024$ ).

\section{Discussion}

Tumor progression is driven by a complex process resulting in an enhanced malignant phenotype. Cancer cells with increased mobility invade adjacent tissues and remodel the extracellular matrix to enter systemic circulation. To acquire mobility, epithelial cancer cells transition into the mesenchymal phenotype via activation of the complex biological program termed EMT. During EMT, epithelial cancer cells lose cell adhesion and polarity and gain 

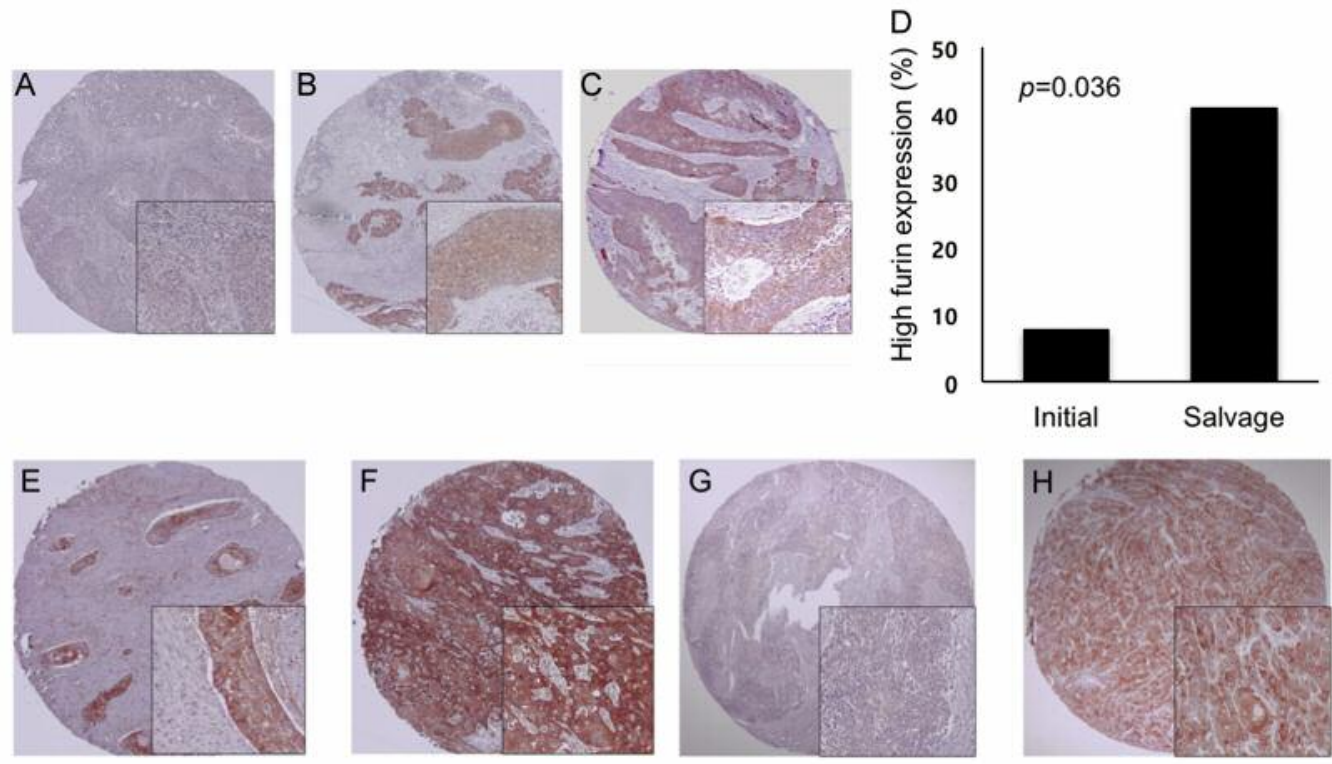

Figure 4. Radiation-induced expression of furin and invasiveness of recurrent laryngeal cancer patients. (A-C) Representative immunohistochemical staining of furin in a laryngeal cancer specimen tissue microarray. Staining scores were obtained by multiplying the staining intensity (0-3) by the staining extent (0-3). Each staining score from A to $C$ was $0(0 \times 0), 6(3 \times 2)$ and $9(3 \times 3)$. Original magnification, 40x; right lower quadrant, 100x. (D) Furin expression in initial specimens and salvage specimens after radiation. Of 22 samples from salvage total laryngectomy and 13 samples from pre-radiation, 9 (40.9\%) and 1 (7.7\%), respectively, showed high furin expression. (E-H) Representative cases of increased furin expression after irradiation. Two representative cases each, showing that the furin staining score increased from 3 to 9 ( $B$ and $C)$ and from 0 to 9 (D and $E)$ after radiation. Original magnification, 40x; right lower quadrant, 100x.

mesenchymal features, including motility and invasiveness. EMT is well-known to be a key step in progression of carcinomas, such as those arising in the head, neck, breast, prostate, ovary and lung (20-22). In this study, after chronic cumulative irradiation, laryngeal cancer cells shifted to EMT. Both types of radioresistant cells studied here showed a spindle-shape appearance in morphology. Furthermore, both AMC-HN-3 and AMC-HN-8 cell lines exhibited an overall up-regulation in the expression of mesenchymal markers after radiation compared to their parental lines, despite differences amongst the individual markers.

Our EMT-programmed cancer cells showed different invasion capacities, despite similar morphologies and molecular profile changes. AMC-HN-3 cells showed enhanced invasion capacity after chronic cumulative irradiation, unlike AMC-HN-8 cells. These individual variations in invasiveness of recurrent laryngeal cancer cells can be explained by the increased expression of furin and concomitant activation of its substrates, MT1-MMP and MMP-2, following irradiation.

MMPs form a family of structurally related zinc-dependent endopeptidases that exist as soluble and membrane-bound enzymes and whose primary function is the breakdown of the extracellular matrix (ECM) and basement membrane, facilitating invasion and metastasis. MMP-2 is believed to be
Table I. Clinical characteristics of salvage total laryngectomy samples in a tissue microarray.

\begin{tabular}{lcc}
\hline Clinical characteristics & $\mathrm{N}=35$ & $\%$ \\
\hline Age, median (range) & $68(48-83)$ & \\
Gender, M/F & $33 / 2$ & $94.3 / 5.7$ \\
Initial stage & & \\
$\quad$ cT, T1/T2/T3 & $20 / 10 / 5$ & $57.1 / 28.6 / 14 / 3$ \\
$\quad$ cN, N0/N1/N2 & $33 / 1 / 1$ & $94.3 / 2.9 / 2.9$ \\
Overall, I/II/III/IV & $20 / 9 / 5 / 1$ & $57.1 / 25.7 / 14.3 / 2.9$ \\
Salvage stage & & \\
rcT, T1/T2/T3/T4 & $0 / 10 / 17 / 8$ & $0 / 28.6 / 48.6 / 22.9$ \\
rcN, N0/N1/N2 & $31 / 2 / 2$ & $88.6 / 5.7 / 5.7$ \\
Overall, I/II/III/IV & $0 / 10 / 16 / 9$ & $0 / 28.6 / 45.7 / 25.7$ \\
Initial treatment & & \\
$\quad$ RTx & 25 & 71.4 \\
CCRTx & 10 & 28.6 \\
Specimen characteristics & & \\
Initial (before RTx or CCRTx) & 3 & 8.5 \\
Salvage (after RTx or CCRTx) & 22 & 62.9 \\
Initial and salvage & 10 & 28.6 \\
\hline
\end{tabular}

RTx, Radiotherapy; CCRTx, concurrent chemoradiotherapy.

a crucial molecule for regulating tumor progression and is a secreted gelatinase that degrades gelatin, laminin and collagen IV $(15,23,24)$. Several malignant tumors broadly 
Table II. Furin expression in recurrent laryngeal cancer tissue microarray who have pre-and post-radiotherapy specimens $(N=10, p=0.024)$.

\begin{tabular}{|c|c|c|c|c|c|c|c|c|}
\hline \multirow[t]{2}{*}{ No } & \multicolumn{2}{|c|}{ Initial } & \multicolumn{2}{|c|}{ Salvage } & \multicolumn{2}{|c|}{ Furin expression } & \multicolumn{2}{|c|}{ Follow-up* } \\
\hline & Stage & Tx & Stage & Tx & Initial & Salvage & Duration (months) & Status \\
\hline 23 & $\mathrm{~T} 2 \mathrm{~N} 0$ & RTx & T2NO & Total Lx & Low & High & 109 & NED \\
\hline 25 & T1N0 & RTx & T4N0 & Total Lx & High & Low & 168 & NED \\
\hline 27 & T1N0 & RTx & T3N0 & Total Lx & Low & High & 14 & $\mathrm{CM}$ \\
\hline 51 & T1N0 & RTx & T4N0 & Total Lx & Low & High & 11 & $\mathrm{CM}$ \\
\hline 52 & T1N0 & RTx & T2N0 & Total Lx & Low & Low & 236 & NED \\
\hline 56 & $\mathrm{~T} 2 \mathrm{~N} 0$ & CCRTx & T3N2 & Total Lx & Low & Low & 125 & NED \\
\hline 61 & $\mathrm{~T} 2 \mathrm{~N} 0$ & RTx & T3N0 & Total Lx & Low & High & 42 & $\mathrm{CM}$ \\
\hline 73 & T3NO & CCRTx & T3NO & Total Lx & Low & High & 138 & NED \\
\hline 81 & T2NO & RTx & T3NO & Total Lx & Low & High & 83 & NED \\
\hline 92 & T1N0 & RTx & T2NO & Total Lx & Low & High & 110 & NED \\
\hline
\end{tabular}

*Follow-up after salvage operation. Tx, Treatment; RTx, radiotherapy; CCRTx, concurrent chemoradiotherapy; Lx, laryngectomy; CM, cancer mortality; NED, no evidence of disease.

overexpress MMP-2; its expression and activity are often associated with tumor aggressiveness and poor prognosis $(15$, 25). In head and neck cancer, MMP-2 is an indicator of lymph node metastasis and a predictor of poor prognosis $(15$, 26-30). Like other MMPs, MMP-2 needs to be processed into an active form as regulated by MT1-MMP and furin. MT1MMP was the first membrane-type metalloproteinase identified to induce specific cleavage of propeptide MMP-2. Furin, the first discovered proprotein convertase, is implicated in proteolytic maturation of proprotein substrates in the secretory pathway. Furin cleaves secretory protein precursors at sites marked by basic amino acids with the RX/RR motif. Hence, MT1-MMP is activated intracellularly by furin before secretion in the Golgi complex (9-11).

In this study, after cumulative irradiation, AMC-HN-3 cells showed increased expression of furin and its substrates, the active forms of MT1-MMP and MMP-2. However, AMC-HN-8 cells did not show a similar increase in expression of these molecules after irradiation. We also found that inhibition of furin activity decreases expression of active MT1-MMP and activation of MMP-2, resulting in inhibition of invasiveness in radioresistant AMC-HN-3 cells.

Our results agree with previous studies investigating furin activity in head and neck cancer cells. Increased furin activity has been shown to correlate with invasiveness and with increased MT1-MMP and subsequent MMP-2 expression in head and neck cancer cells. Further, head and neck cancer cells showed decreased expression of MT1-MMP and MMP2 and decreased invasion potential in the presence of the furin inhibitor CMK $(12,13)$. Studies investigating furin activity in other types of cancers, including human astrocytoma, colon cancer and fibrosarcoma cells, also reported that furin inhibitors decreased invasion potential and MT1-MMP expression $(10,31,32)$.
The mechanism underlying changes in furin expression in response to radiation is unclear and further investigations are warranted. However, these observations have important clinical implications. Furin may be used as a biomarker for predicting clinical behavior after radiotherapy in recurrent laryngeal cancer. In this study, augmented furin may also correspond to aggressiveness of recurrent laryngeal cancer in patients. Furin expression was higher in salvage samples obtained after radiation than in pre-radiation samples. Among 10 patients with both pre- and post-radiation samples, furin expression was increased in 7 patients after radiation, with all-cancer mortality (3 patients) being observed in this patients' group. Indeed, furin is often up-regulated in cancer and has recently been associated with the aggressive cancer phenotype $(9-11,14)$.

In summary, our study provides evidence that furin activity and EMT are potential indicators of the aggressive phenotype in recurrent laryngeal cancer after irradiation and that furin is a potentially useful target against laryngeal cancer.

\section{Conflicts of Interest}

No potential conflicts of interest are disclosed.

\section{Acknowledgements}

This research was supported by the Basic Science Research Program through the National Research Foundation of Korea (NRF) funded by the Ministry of Science, ICT and Future planning (NRF2015R1A2A1A01003050) and a grant from the Bio and Medical Technology Department Program of the National Research Foundation funded by the Korean government (MEST) (NRF2012M3A9B2028788). 


\section{References}

1 Argiris A, Karamouzis MV, Raben D and Ferris R: Head and neck cancer. Lancet 371: 1695-1709, 2008.

2 Cooper JS, Pajak TF, Forastiere AA, Jacobs J, Campbell BH, Saxman SB, Kish JA, Kim HE, Cmelak AJ, Rotman M, Machtay M, Ensley JF, Chao KS, Schultz CJ, Lee $\mathrm{N}$ and $\mathrm{Fu} \mathrm{KK}$; Radiation Therapy Oncology Group 9501/Intergroup: Postoperative concurrent radiotherapy and chemotherapy for high-risk squamous-cell carcinoma of the head and neck. N Engl J Med 350: 1937-1944, 2004.

3 The Department of Veterans Affairs Laryngeal Cancer Study Group: Induction chemotherapy plus radiation compared with surgery plus radiation in patients with advanced laryngeal cancer. N Engl J Med 324: 1685-1690, 1991.

4 Gilbert $\mathrm{H}$ and Kagan AR: Recurrence patterns in squamous cell carcinoma of the oral cavity, pharynx, and larynx. J Surg Oncol 6: 357-380, 1974.

5 Sacco AG and Cohen EE: Current treatment options for recurrent or metastatic head and neck squamous cell carcinoma. J Clin Oncol 33: 3305-3313, 2015.

6 Vermorken JB, Mesia R, Rivera F, Remenar E, Kawecki A, Rottey S, Erfan J, Zabolotnyy D, Kienzer HR, Cupissol D, Peyrade F, Benasso M, Vynnychenko I, De Raucourt D, Bokemeyer C, Schueler A, Amellal N and Hitt R: Platinumbased chemotherapy plus cetuximab in head and neck cancer. N Engl J Med 359: 1116-1127, 2008.

7 Argiris A, Karamouzis MV, Gooding WE, Branstetter BF, Zhong S, Raez LE, Savvides P and Romkes M: Phase II trial of pemetrexed and bevacizumab in patients with recurrent or metastatic head and neck cancer. J Clin Oncol 29: 1140-1145, 2011.

8 Lee JC, Lee WH, Min YJ, Cha HJ, Han MW, Chang HW, Kim SA, Choi SH, Kim SW and Kim SY: Development of TRAIL resistance by radiation-induced hypermethylation of DR4 CpG island in recurrent laryngeal squamous cell carcinoma. Int $\mathrm{J}$ Radiat Oncol Biol Phys 88: 1203-1211, 2014.

9 Seidah NG and Prat A: The biology and therapeutic targeting of the proprotein convertases. Nat Rev Drug Discov 11: 367-383, 2012 .

10 Scamuffa N, Siegfried G, Bontemps Y, Ma L, Basak A, Cherel G, Calvo F, Seidah NG and Khatib AM: Selective inhibition of proprotein convertases represses the metastatic potential of human colorectal tumor cells. J Clin Invest 118: 352-363, 2008.

11 Couture F, Kwiatkowska A, Dory YL and Day R: Therapeutic uses of furin and its inhibitors: a patent review. Expert Opin Ther Pat 25: 379-396, 2015.

12 Bassi DE, Mahloogi H, Al-Saleem L, Lopez De Cicco R, Ridge JA and Klein-Szanto AJ: Elevated furin expression in aggressive human head and neck tumors and tumor cell lines. Mol Carcinog 31: 224-232, 2001.

13 Bassi DE, Mahloogi H, Lopez De Cicco R and Klein-Szanto A: Increased furin activity enhances the malignant phenotype of human head and neck cancer cells. Am J Pathol 162: 439-447, 2003.

14 Bassi DE, Fu J, Lopez de Cicco R and Klein-Szanto AJ: Proprotein convertases: "master switches" in the regulation of tumor growth and progression. Mol Carcinog 44: 151-161, 2005.

15 Chien MH, Lin CW, Cheng CW, Wen YC and Yang SF: Matrix metalloproteinase-2 as a target or head and neck cancer therapy. Expert Opin Ther Targets 17: 203-216, 2013.
16 Kessenbrock K, Plaks V and Werb Z. Matrix Metalloproteinases: Regulators of the tumor microenvironment. Cell 141: 52-67, 2010.

17 Page-McCaw A, Ewald AJ and Werb Z: Matrix metalloproteinases and the regulation of tissue remodelling. Nat Rev Mol Cell Biol 8: 221-233, 2007.

$18 \mathrm{Kim} \mathrm{SY,} \mathrm{Chu} \mathrm{KC,} \mathrm{Lee} \mathrm{HR,} \mathrm{Lee} \mathrm{KS} \mathrm{and} \mathrm{Carey} \mathrm{TE:}$ Establishment and characterization of nine new head and neck cancer cell lines. Acta Otolaryngol (Stockh) 117: 775-784, 1997.

19 Lee JC, Lee WH, Min YJ, Cha HJ, Han MW, Chang HW, Kim SA, Choi SH, Kim SW and Kim SY: Development of TRAIL resistance by radiation-induced hypermethylation of $\mathrm{dr} 4 \mathrm{cpg}$ island in recurrent laryngeal squamous cell carcinoma. Int. J Radiat Oncol 88: 1203-1211, 2014.

20 Polyak K and Weinberg RA: Transitions between epithelial and mesenchymal states: acquisition of malignant and stem cell traits. Nat Rev Cancer 9: 265-273, 2009.

21 Thiery JP, Acloque H, Huang RY and Nieto MA: Epithelialmesenchymal transitions in development and disease. Cell 139: 871-890, 2009.

22 Nieto MA: The ins and outs of the epithelial to mesenchymal transition in health and disease. Annu Rev Cell Dev Biol 27: 347-376, 2011.

23 Klein T and Bischoff R: Physiology and pathophysiology of matrix metalloproteases. Amino Acids 41: 271-290, 2011.

24 Page RE, Klein-Szanto AJ, Litwin S, Nicolas E, Al-Jumaily R, Alexander P, Godwin AK, Ross EA, Schilder RJ and Bassi DE: Increased expression of the pro-protein convertase furin predicts decreased survival in ovarian cancer. Cell Oncol 29: 289-299, 2007.

25 Roy R, Yang J and Moses MA: Matrix metalloproteinases as novel biomarkers and potential therapeutic targets in human cancer. J Clin Oncol 27: 5287-5297, 2009.

26 Patel BP, Shah SV, Shukla SN, Shah PM and Patel PS: Clinical significance of MMP-2 and MMP-9 in patients with oral cancer. Head Neck 29: 564-572, 2007.

27 Chung CH: Molecular classification of head and neck squamous cell carcinomas using patterns of gene expression. Cancer Cell 5: 489-500, 2004.

28 Bogusiewicz M, Stryjecka-Zimmer M, Szymanski M, Rechberger $\mathrm{T}$ and Golabek $\mathrm{W}$ : Activity of matrix metalloproteinases-2 and -9 in advanced laryngeal cancer. Otolaryngol Head Neck Surg 128: 132-136, 2003.

29 Xie M, Sun Y and Li Y: Expression of matrix metalloproteinases in supraglottic carcinoma and its clinical implication for estimating lymph node metastases. Laryngoscope 114: 2243-2248, 2004.

30 Wiegand S, Dünne AA, Müller HH, Mandic R, Barth P, Davis RK and Werner JA: Meta-analysis of the significance of matrix metalloproteinases for lymph node disease in patients with head and neck squamous cell carcinoma. Cancer 104: 94-100, 2005.

31 Coppola JM, Bhojani MS, Ross BD and Rehemtulla A: A smallmolecule furin inhibitor inhibits cancer cell motility and invasiveness. Neoplasia 10: 363-370, 2008.

32 Mercapide J, Lopez De Cicco R, Bassi DE, Castresana JS, Thomas G and Klein-Szanto AJ: Inhibition of furin-mediated processing results in suppression of astrocytoma cell growth and invasiveness. Clin Cancer Res 8: 1740-1746, 2002.

Received August 12, 2016

Revised September 6, 2016 Accepted September 26, 2016 